OPEN ACCESS

Edited by:

Li Cai,

Rutgers, The State University

of New Jersey, United States

Reviewed by:

Aiko Sada,

Kumamoto University, Japan

Delin Mo,

Sun Yat-sen University, China

*Correspondence:

Xiangdong Ding

xding@cau.edu.cn

Specialty section:

This article was submitted to

Morphogenesis and Patterning,

a section of the journal

Frontiers in Cell and Developmental

Biology

Received: 08 June 2021

Accepted: 13 September 2021

Published: 07 October 2021

Citation:

Jiang Y, Zou Q, LiU B, Li S,

Wang Y, Liu T and Ding X (2021) Atlas

of Prenatal Hair Follicle

Morphogenesis Using the Pig as

a Model System.

Front. Cell Dev. Biol. 9:721979.

doi: 10.3389/fcell.2021.721979

\section{Atlas of Prenatal Hair Follicle Morphogenesis Using the Pig as a Model System}

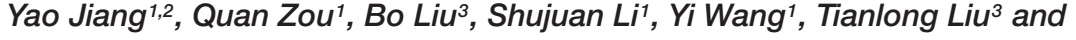 \\ Xiangdong Ding ${ }^{1 *}$
}

${ }^{1}$ National Engineering Laboratory for Animal Breeding, Laboratory of Animal Genetics, Breeding and Reproduction, Ministry of Agriculture, College of Animal Science and Technology, China Agricultural University, Beijing, China, ${ }^{2}$ Institute of Animal Husbandry and Veterinary Medicine, Anhui Academy of Agricultural Sciences, Hefei, China, ${ }^{3}$ Key Laboratory of Animal Epidemiology of the Ministry of Agriculture, College of Veterinary Medicine, China Agricultural University, Beijing, China

The pig is an increasingly popular biomedical model, but only a few in depth data exist on its studies in hair follicle (HF) morphogenesis and development. Hence, the objective of this study was to identify the suitability of the pig as an animal model for human hair research. We performed a classification of pig HF morphogenesis stages and hair types. All four different hair types sampled from 17 different body parts in pig were similar to those of human. The Guard_2 sub-type was more similar to type II human scalp hair while Guard_1, Awl, Auchene, and Zigzag were similar to type I scalp hair. Based on morphological observation and marker gene expression of HF at 11 different embryonic days and six postnatal days, we classified pig HF morphogenesis development from E41 to P45 into three main periods - induction (E37-E41), organogenesis (E41-E85), and cytodifferentiation (>E85). Furthermore, we demonstrated that human and pig share high similarities in HF morphogenesis occurrence time (early/mid gestational) and marker gene expression patterns. Our findings will facilitate the study of human follicle morphogenesis and research on complex hair diseases and offer researchers a suitable model for human hair research.

Keywords: pig, hair follicle, hair types, morphogenesis development, animal model

\section{INTRODUCTION}

Hair follicles (HFs) are one of the most important appendages of the skin. Their development is divided into prenatal HF morphogenesis development and postnatal HF cycle development (Schneider et al., 2009). HF morphogenesis development in the prenatal stage is critically important, which determines HF cycle development in the postnatal stage. HF morphogenesis has been summarized into three distinct periods - induction (stage $0-1$ ), organogenesis (stage 2-5)

Abbreviations: HF, hair follicle; DC, dermal condensate; DP, dermal papilla; IRS, inner root sheath; ORS, outer root sheath; SG, sebaceous gland; CTS, connective tissue sheath; AP, alkaline phosphatase activity; IL-1-RI, interleukin-1 receptor type 1; TGF- $\beta$-RII, transforming growth factor $\beta$ receptor type II; SOX2, SRY-box transcription factor 2 ; LEF1, lymphoid enhancer binding factor 1; SOX9, SRY-box transcription factor 9; KRT15, keratin 15; Wnt5a, Wnt family member 5A; CTNNB1, catenin beta 1; DKK4, Dickkopf WNT signaling pathway inhibitor 4; BMP4, bone morphogenetic protein 4. 
and cytodifferentiation (stage 6-8) based on their key characteristics (Paus et al., 1999). Several classical genetic markers such as SOX2 (Saxena et al., 2019), LEF1 (Boras-Granic et al., 2006), SOX9 (Nguyen et al., 2018), and KRT15 (Miyachi et al., 2018) have been established as critical genes involved in each step of morphogenesis, and studies on these genes have enabled a fine-toothed dissection of the cellular and molecular dynamics of HF morphogenesis (Sennett et al., 2015; Tomann et al., 2016).

To date, morphological and development studies that also address the genetic and molecular mechanisms underlying human HF development and structure have been conducted using mouse models providing a deeper understanding of the numerous abnormal phenotypes of the HF (Duverger and Morasso, 2014; Fuchs, 2016). These studies have, however, identified fundamental differences in the morphological structure (van Ravenzwaay and Leibold, 2004), developmental mechanisms (Porter, 2003; Duverger and Morasso, 2009; Pratt et al., 2017), and hair diseases (Porter, 2003) between human and mouse HFs. The shortage of human embryos and the ethical issues surrounding their use for research purposes have also restricted further study on human hair follicle morphogenesis during embryogenesis. Considering the above-mentioned draw-backs, a new animal model with easy availability and a high similarity to the human HF development is required to facilitate hair follicle morphogenesis research.

One such potential model, the pig, has emerged in recent years as a model for the study of numerous diseases such as atherosclerosis (Jensen et al., 2010), diabetes (Renner et al., 2010), cystic fibrosis (Rogers et al., 2008), retinitis pigmentosa (Ross et al., 2012), and cardiovascular disease (Tsang et al., 2016). A high similarity has been noted between human and pig with regard to anatomy, genetics, and physiology, and the pig is being increasingly used as an alternative research model to rodents, canines, or non-human primates (Meurens et al., 2012; Pabst, 2020; Zuidema and Sutovsky, 2020). However, very limited data are available regarding the morphogenesis and development of pig hair follicles, despite pig skin showing several anatomical and physiological similarities with human skin (Liu et al., 2010; Turner et al., 2015). Therefore, further studies are needed with regard to the suitability of using the pig as an animal model for human HF.

Thus, the objectives of this study were to evaluate the possibility of using the pig as an animal model for human HF research. In this study we have: (1) established accurate classification and recognition of HF morphogenesis stages in pig embryonic development; (2) compared morphological structures and the expression of marker genes of the specialized hair follicle of pig with human and mouse HF; and (3) classified the different hair types present in the pig skin.

\section{MATERIALS AND METHODS}

\section{Ethics Approval}

All animal procedures were evaluated and authorized by Institutional Animal Care and Use Committee (IACUC). The whole procedure for samples collected was carried out in strict accordance with the protocol approved by the IACUC at the China Agricultural University. The IACUC of the China Agricultural University specifically approved this study (permit number DK996).

\section{Pig Skin Samples From Embryonic and Postnatal Stages}

Yorkshire pig skin samples $(1 \times 3 \mathrm{~cm})$ of 11 different embryonic (E) (E37, E39, E41, E43, E45, E52, E60, E85, E103, E107, E113) and six postnatal (P) days (P5, P11, P19, P22, P26, P45) were obtained from a well-defined dorsal skin region precisely located as described in the operation manual of Paus et al. (1999).

\section{Phenotype Measurement of Hair Shafts}

Three piglets (P30) were used to perform phenotype measurement analysis. Hair shafts were sampled from 17 different body parts (Figure 1A): back (upper, middle and bottom), forelimb, hindlimb, epigastrium, hypogastrium, forehoof, hind hoof, ear marginal, head, eyebrow, eyelash, whiskers (mouth and jaw), shoulder, and buttocks. Except for the eyebrow, eyelash, and whiskers (mouth and jaw), where all the hair shafts were collected, hair shafts from the other 13 body parts within $1 \mathrm{~cm}^{2}$ region were collected, and three replicates were made for each body part. For each part, hair shafts were measured for hair length $(\mathrm{mm})$ by a straight ruler and for hair thickness $(\mu \mathrm{m})$ and hair density $\left(/ \mathrm{cm}^{2}\right)$ by light microscopy.

Three randomly selected images were used to perform analysis.

\section{Classification of Hair Types}

In accordance with the classification of mouse hair types (Duverger and Morasso, 2009), pig hair shafts were classified into different hair types and summarized with length, number of waves, and bends as criteria.

\section{Identification of Hair Follicle Morphogenesis Development Stages}

As described by Paus et al. (1999), the HF morphogenesis development stages were identified based on the characteristic derivatives of different time periods - hair placode for induction (stage 1); hair germ, DC for stage 2; hair peg, DP for stages 3-5 in organogenesis; IRS, ORS for stages 6-7; and hair shaft for stage 8 in cytodifferentiation. Hematoxylin and eosin (H\&E) staining was performed to visualize the characteristic derivatives of each stage. Immunofluorescence of marker gene expression at different stages was also performed for the distinction of the different HF morphogenesis stages. Five antibodies used are listed in Table 1, and negative control (fetal bovine serum substitute antibody) result, in Supplementary Figure 1.

All dorsal skin samples were embedded in paraffin, sectioned at $4 \mu \mathrm{m}$ for the transverse and longitudinal sections. The protocol for the immunofluorescence and H\&E (Sigma-Aldrich, St. Louis, MO, United States) staining was as described in Nguyen et al. (2018). Sections were imaged using the TCS SP5 
confocal microscope (Leica, Wetzlar, Germany) using $\times 10, \times 20$, or $\times 40$ objectives.

\section{RESULTS}

\section{Classification and Recognition of Hair Types in Pig}

As shown in Figure 1B, four different hair types - Awl, Guard, Auchene, and Zigzag - present in the mouse could also be found in pig. The upper, middle, and bottom of back, epigastrium, and hypogastrium, collectively called the Back region, shared the same hair types. Among the four hair types, Guard (divided into Guard_1/Guard_2, discussed later) was the most abundant (32-100\%) amongst all hair types, while Auchene and Zigzag only represented $4-10 \%$ and $2-8 \%$ (except $52 \%$ Zigzag hair on head) in different body parts. All four hair types were identified on the back, limb, hoof and shoulder, while buttocks, head, eyebrow, and beard contained two to three hair types; the ear and eyelash only contained Guard and Awl hair, respectively.

The quantification of $\mathrm{HF}$ length and HF thickness in the four hair types in pig was significantly different (Figure 1C). Comparing the two subtypes of Guard hair with the other hair types revealed that Guard_1 had the most length (41.074 $\pm 4.945 \mathrm{~mm}, p<0.001)$ and thickness $(37.895 \pm 3.423 \mu \mathrm{m}, p<0.001)$, Guard_2 was the shortest $(27.857 \pm 5.201 \mathrm{~mm}, p<0.001)$, and Zigzag had the least thickness $(22.909 \pm 4.300 \mu \mathrm{m}, p<0.001)$. The hair density of the head $\left(49.333 \pm 3.512 / \mathrm{cm}^{2}\right)$ and back $\left(45.667 \pm 2.082 / \mathrm{cm}^{2}\right)$ was significantly higher $(p<0.01)$ than that of the other body parts (Figure 1D). No significant difference in HF density was found between the limb $\left(14.000 \pm 1.000 / \mathrm{cm}^{2}\right)$ with shoulder $\left(17.333 \pm 2.517 / \mathrm{cm}^{2}\right)$ and hoof $\left(23.667 \pm 3.215 / \mathrm{cm}^{2}\right)$ with $\left(25.000 \pm 1.732 / \mathrm{cm}^{2}\right)$.

\section{Identification of Distinct Stages in Pig Hair Follicle Morphogenesis}

Hair follicle morphogenesis development was observed at 11 different embryonic (E) and six postnatal (P) days (Supplementary Figure 2). According to the characteristic derivatives and expression of marker genes in different regions of the $\mathrm{HF}$ at different time points, pig $\mathrm{HF}$ morphogenesis development started from E37 and ended at P45. Eight key time points (E37, E41, E52, E60, E85, E107, P2, and P45) were selected to explore additional details of HF morphogenesis development and are described below.

Undifferentiated (Stage 0, $\leq$ E37) Figure 2 indicates that the first stage of HF morphogenesis development of pig occurs before

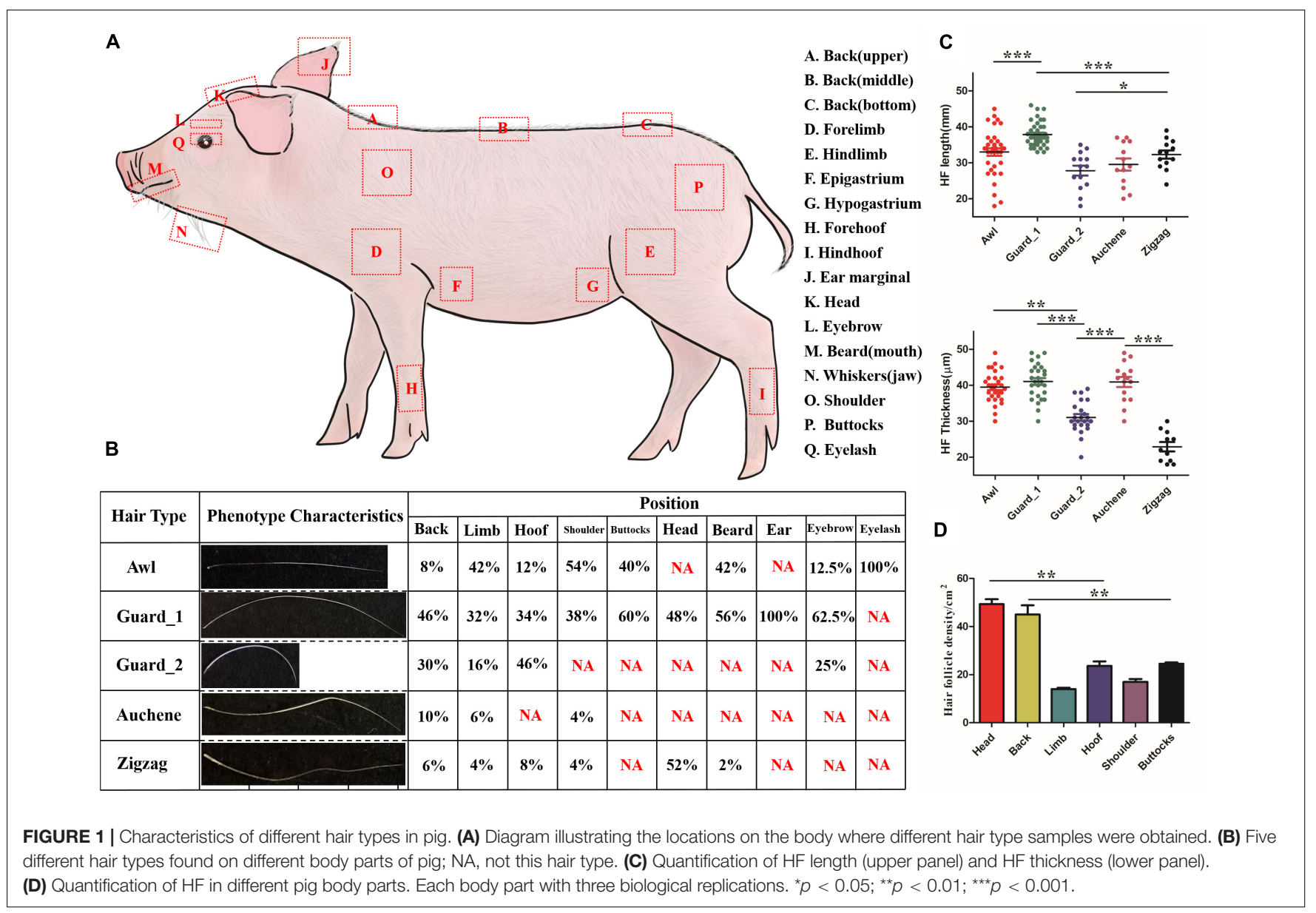


TABLE 1 | The information of five antibodies in this study.

\begin{tabular}{|c|c|c|c|c|c|c|}
\hline \multirow{2}{*}{$\begin{array}{l}\text { Periods } \\
\text { Stage }\end{array}$} & \multirow{2}{*}{$\begin{array}{c}\text { Undifferentiated } \\
\text { Stage } 0\end{array}$} & \multirow{2}{*}{$\frac{\text { Induction }}{\text { Stage } 1}$} & \multirow{2}{*}{$\begin{array}{c}\text { Organogenesis } \\
\text { Stages 3-5 }\end{array}$} & \multicolumn{3}{|c|}{ Cytodifferentiation } \\
\hline & & & & Stages 6-7 & \multicolumn{2}{|c|}{ Stage 8} \\
\hline Antibody & LEF1 & & LEF1/SOX2 & sox9 & K15 & Ki67 \\
\hline $\begin{array}{l}\text { Expression } \\
\text { region }\end{array}$ & $\begin{array}{l}\text { Basal keratinocytes } \\
\text { hair placode }\end{array}$ & $\begin{array}{c}\text { Hair } \\
\text { placode }\end{array}$ & DC/DP & IRS, ORS, hair shaft & Hair shaft, ORS & Proliferation cells \\
\hline \multirow[t]{2}{*}{ Parameter } & \multicolumn{2}{|c|}{ Abcam, ab137872 } & Bioss, bs-23177R & Abcam, ab185966 & Santa, sc-47697 & CST, 9449T \\
\hline & \multicolumn{2}{|l|}{$1: 500$} & $1: 500$ & $1: 800$ & $1: 500$ & $1: 1,000$ \\
\hline Reference & \multicolumn{2}{|c|}{$\begin{array}{c}\text { Human (Gay et al., 2015) } \\
\text { Mouse (Telerman et al., 2017) }\end{array}$} & $\begin{array}{l}\text { Mouse (Saxena et al., } \\
\text { 2019) }\end{array}$ & $\begin{array}{c}\text { Human (Gay et al., 2015) } \\
\text { Mouse (Fantauzzo et al., 2012) }\end{array}$ & $\begin{array}{l}\text { Human (Yang et al., 2014; } \\
\text { Purbal, Haslam et al., 2015) }\end{array}$ & Mouse (Liu et al., 2019) \\
\hline
\end{tabular}

DAPI (Abcam, ab104139) for nuclear, secondary antibodies coupled to Alexa488 (Abcam, ab150077, 1:200), Alexa549 (Abcam, ab150116, 1:200), or Alexa647 (Abcam, ab150077, 1:200).

E37, also termed as pre-germ stage. Figure 2A demonstrates a schematic diagram of the characteristic structures of the skin and HFs in this undifferentiated stage. The epidermis and dermis of skin were sharply demarcated, and basal epidermal cells were in a uniform layer. No morphological signs or molecular distinction patterns of HF were recognized in the skin (Figures 2B,C). Only LEF1 was strongly expressed in epidermal keratinocytes in the basal and suprabasal cell layers of the epidermis (Figure 2D).

Induction (Stage 1, E41-E52) Stage 1 of HF morphogenesis is also termed as the induction stage. The representative characteristic structures of this stage are the formation of the hair placode (Figure 2E). Our results demonstrate that stage 1 of pig HF morphogenesis took place at around E41; the epidermal keratinocytes assembled in an upright position, and started proliferating and thickening to form the hair placode, as illustrated in Figures 2F,G. The hair placode showed strong expression of LEF1, whereas no expression was deleted in dermal fibroblasts (Figure $\mathbf{2 H}$ ). Additionally, another less obvious characteristic of HF development in stage 1 was a localized increase in the number of dermal fibroblasts under the hair placode (Figure 2G). These fibroblasts began to display a slightly altered orientation, as a preparation for their subsequent aggregation.

Organogenesis (Stages 2-5, E52-E85) The organogenesis stage of HF morphogenesis is usually recognized based on the characteristic structure of hair germ and dermal condensate (DC) in stage 2 (Figure 2I), and hair peg and DP at stages 3-5 (Figure 2N). Stage 2 occurred at E52 as illustrated in Figures 2J,K. At this stage, the hair placode became more prominent and enlarged with a broad column called hair germ, which was primarily a consequence of massive epithelial cell proliferation in a well circumscribed patch of basal layer epidermal keratinocytes (Figure 2J). Few mesenchymal cells in the dermis were found condensed into a cap-like condensation and finally formed the DC (Figure 2K). LEF1 and SOX2 genes were strongly expressed in the enlarged epithelial hair germ and DC, respectively (Figures $2 \mathbf{L}, \mathbf{M}$ ).

Stages 3-5 were found to be the continuation of stage 2, and the HF morphogenesis in these three stages happened at around E60-E85. Based on the characteristic structures of $\mathrm{HF}$, at this stage, hair germ and DC had differentiated into hair peg and dermal papilla (DP) (Figure 2N). The hair peg is generally identified by large, tightly packed vertically oriented keratinocytes which directly invaginate into the dermis (Figure 2O). The DC remains on the leading edge of the downgrowing hair peg and, upon engulfment by the HF matrix, transitions into a bulb-like DP (Figure 2P). Like the previous stages, LEF1 and SOX2 were more strongly expressed on hair peg and DP, respectively (Figures 2Q,R).

Cytodifferentiation (Stages $\mathbf{6 - 8},>$ E85) In this period, the mature HF was almost developed while inner root sheath (IRS), outer root sheath (ORS), and hair shaft were visible (Figures 3A,F). In stages 6-7 (E85-E107), the hair peg resembled a mature HF more closely. The DP was completely engulfed by the surrounding matrix cells at the base of the hair peg, the IRS elongated up through the developing follicle, and the hair canal development had started (Figure 3B). The hair shaft, IRS, and ORS were visible in transverse section (Figure 3C). Moreover, the mature ORS and hair shaft were recognized by SOX9-stained hair keratins, while the IRS, derived from DP, showed almost negative expression (Figures 3D,E).

In stage 8 ( $>$ E107), the HF had already matured, and the key characteristic structures were hair canal visibility and the hair shaft formation. The tip of the hair shaft exited the IRS, entered the hair canal, and emerged through the epidermis (Figure 3G). The hair shaft, IRS, ORS, and CTS were all clearly visible in transverse sections (Figure $3 \mathbf{H}$ ). The expression of K15 was detected in the ORS (Figure 3I) and hair shaft whose precursor cells coming from epidermis basal keratinocytes had strong expression of SOX9 (Figure 3J). Morphologically, the HF was mature and resembled an early-stage anagen $\mathrm{HF}$.

\section{Prenatal Hair Follicle Morphogenesis Development Maintained Until Postnatal P45}

According to our observations of $\mathrm{HFs}$ at several periods after E107 (Supplementary Figure 1), HF morphogenesis development was maintained until P45, and then the first stage of HF cycle development commenced - the catagen stage. The period of E107-P45 resembled a pseudo-anagen stage as the hair follicle showed continued development and the characteristic morphology was similar to anagen V in P5 (Figure 4A). In this 
stage, the tip of the hair shaft grew through the hair canal and epidermis as seen in longitudinal section (Figure 4B) while the connective tissue sheath (CTS), ORS, IRS, and hair shaft formed a cylinder from outside to inside in transverse section (Figure 4D). In addition, the sebaceous gland (SG) had located on the side of the HF (Figure 4C). As the indicator of proliferation ability of keratinocyte cells, the expression of SOX9 and Ki67 at P5 was reduced, compared with their expression at E107 (Figures 4E,F), indicating the slowing down of HF morphogenesis development and gradual entry into the final stages of development.

At P45, the key characteristic structure of catagen period is the beginning of atrophy of the DP to a ball-shaped structure

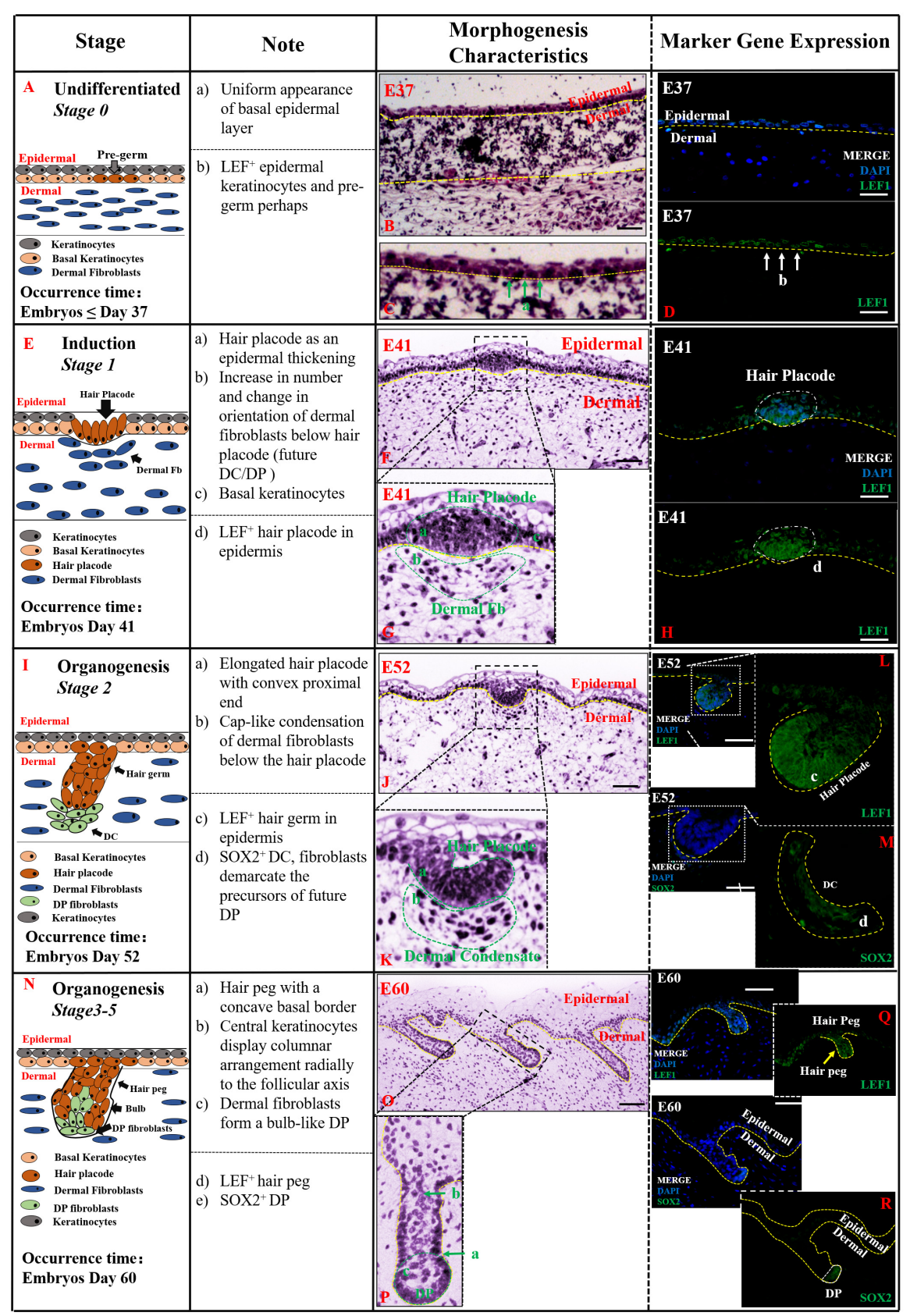

FIGURE 2 | The identification stage 0 (Undifferentiated), stage 1 (Induction), and stage 2-5 (Organogenesis) in pig hair follicle morphogenesis. A schematic diagram of HF morphogenesis in stage 0 at E37 (A), stage 1 between E41 and E52 (E), stage 2 (E52-E60) (I), and stages 3-5 (E60-E85) (N) between E52 and E85. The Note column summarizes simple basic parameters for HF staging by H\&E staining (above dotted line), and marker gene expression (below dotted line). The characteristic of pig hair follicle morphology by H\&E at E37 (B), E41 (F), E52 (J), and E60 (O). (C,G,K,P) Partial enlarged detail of hair follicle in parts (B,F,J,O), respectively; LEF1 expression in pig hair follicle morphology at E37 (D), E41 (H), E52 (L), and E60 (Q) by IF; Sox2 expression in pig DC at E52 (M) and pig DP at E52 (R) by IF. Green and white arrows represent basal keratinocytes; yellow arrows represent hair peg. Scale bars: $50 \mathrm{~mm}$. 


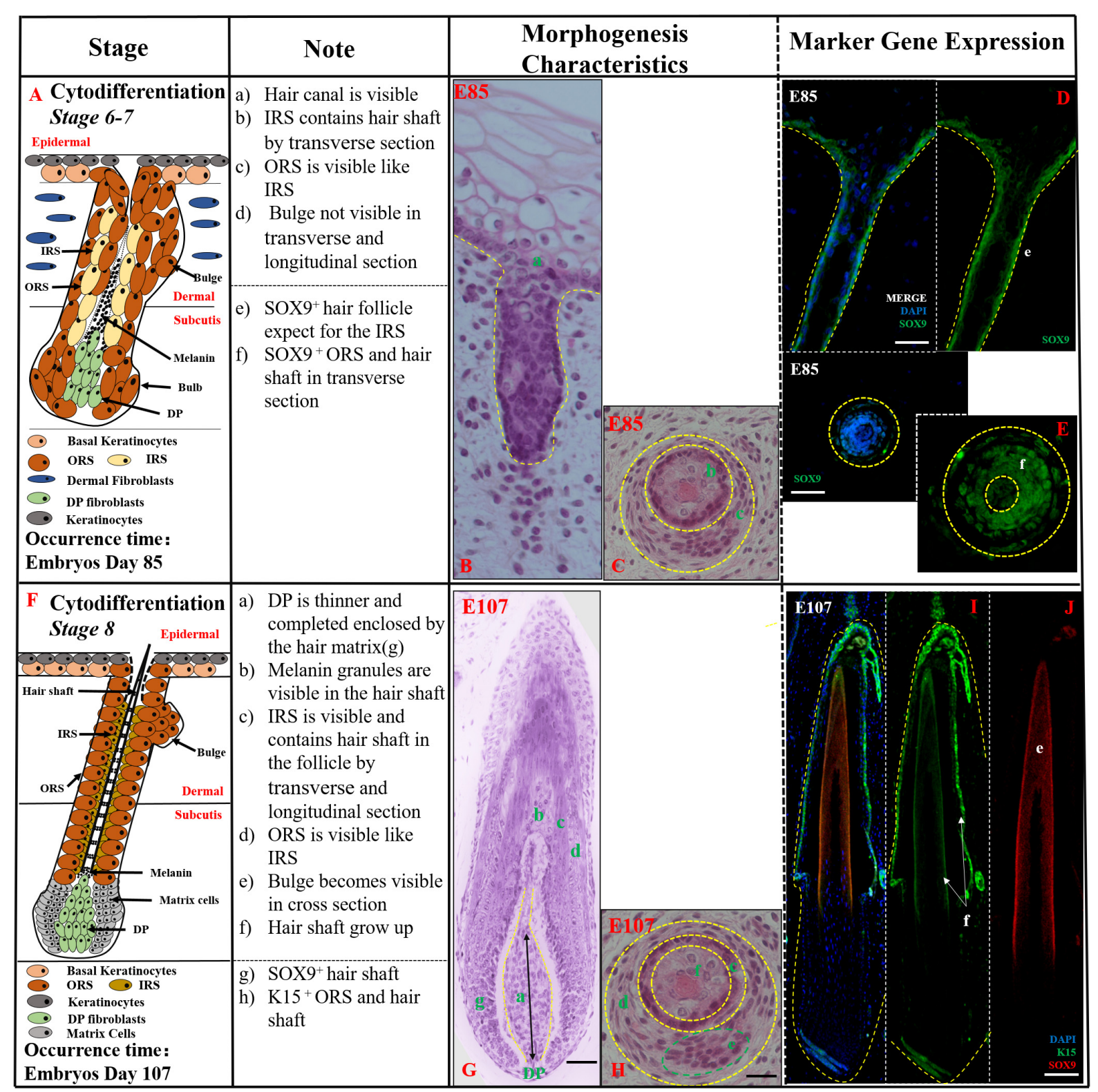

FIGURE 3 | The identification of stages 6-8 (Cytodifferentiation) of pig hair follicle morphogenesis. (A,F) A schematic diagram of stages 6-7 and stage 8 in HF morphogenesis after E85. The Note column summarizes simple basic parameters for HF stage by H\&E staining (above dotted line) and marker gene expression (below dotted line). (B) The characteristics of pig hair follicle morphology at E85 by H\&E. (C) Partial enlarged detail of hair follicle in part (B); SOX9 expression in ORS and hair shaft at E85 in longitudinal (D) and transverse (E) section by IF. (G) The characteristics of pig hair follicle morphology at E107 by H\&E. (H) Partial enlarged detail of hair follicle in part (G). (I) K15 expression in pig ORS and hair shaft at E107 by IF. (J) Sox9 expression in pig hair shaft at E107 by IF. Scale bars: 50 mm.

(Figure 4G). As shown in Figures 4H,I, HF length at P45 was significantly shorter than in pseudo anagen $\mathrm{V}$, closer to the epidermis and ORS forming the germ capsule around the club hair. The HF displayed another catagen characteristic, the presence of an epithelial strand between the secondary germ capsule and the DP. Furthermore, no SOX9 expression was detected on the hair shaft (Figure 4K) and strong expression of Ki67 was demonstrated in the HF (Figure 4J), indicating that the HF was inactive and had entered the catagen stage. Interestingly, the characteristic of pseudo-anagen IIIb stage was also detected at this stage; the newly formed hair shaft was fully enclosed by the IRS, while the older hair shaft had moved up. Furthermore, strong expression of SOX9 (Figure 4M) and Ki67 (Figure 4L) in ORS and contraction of matrix cells were identified, suggesting the commencement of the first pseudoanagen period in HF morphogenesis.

\section{DISCUSSION}

Although the mouse is the most commonly used animal model for research on human HF development, the classification of hair 


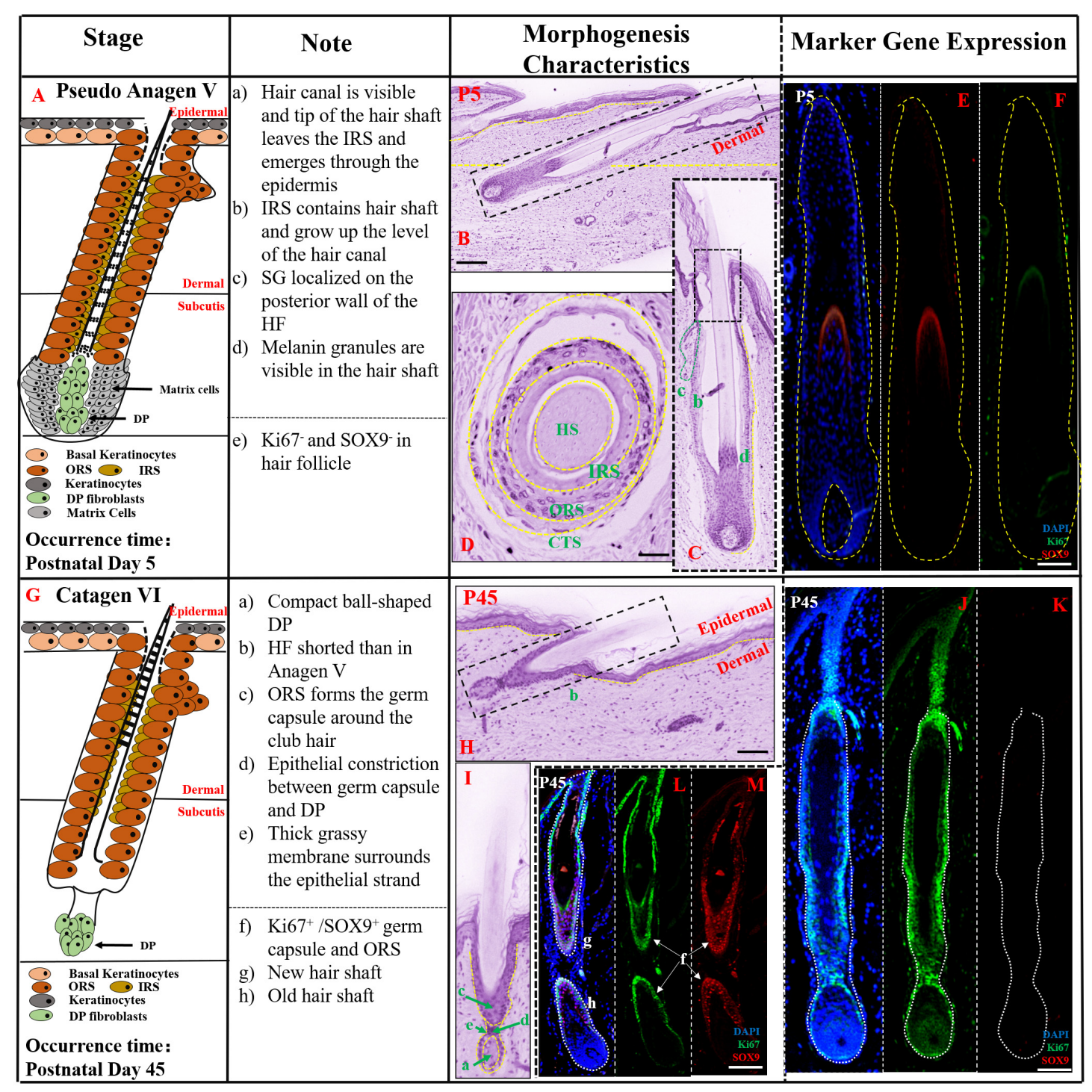

FIGURE 4 | The prenatal hair follicle morphogenesis development maintained until postnatal P45. (A,G) A schematic diagram of HF morphogenesis development in the postnatal stage (P5-P45). The Note column summarizes simple basic parameters for HF staging by H\&E staining (above dotted line) and marker gene expression (below dotted line). (B) The characteristics of pig hair follicle morphology at P5 by H\&E. (C) Partial enlarged detail of hair follicle in part (B). The characteristics of pig hair follicle morphology in transverse section at P5 (D) and P45 (H) by HE; (E) SOX9 expression in hair shaft at P5 by IF; (F) Ki67 expression in proliferation keratinocyte cells at P5 by IF; (I) Partial enlarged detail of hair follicle in part (H). (J,L) Ki67 expression in pig proliferation keratinocytes cells at P45 by IF. (K,M) Sox9 expression in pig hair follicle at P45 by IF. Scale bars: $50 \mathrm{~mm}$.

types in human has been implemented in different ways, mainly depending on the body parts (e.g., scalp, beard, chest, pubic, and axillary hair) with scalp hair being the most studied hair type (Duverger and Morasso, 2009; Buffoli et al., 2014). Nonetheless, similarities could still be explored between pig and human scalp hair. De la Mettrie et al. (2007) collected hair samples from 18 countries and classified eight main types of scalp hair based on curve diameter (CD), waves (w), and twists (i). In this study, all four different hair types were identified in pig referred to mouse. However, based on the hair shaft length and diameter, Guard hair in pig was further divided into two different subtypes of Guard_1 and Guard_2. Guard_2 had a larger angle bending and shorter length. In comparison with the CD and w of human scalp hair (Figure 5A), the CDs of pig Awl, Auchene, and Zigzag hair types were greater than 20 and the CD of Guard_1 was 10.5-15. Besides, they were similar with Type I human scalp hair reported by De la Mettrie et al. (2007), while the CD of Guard_2 was 5.8-10.5 like Type II in human scalp hair (Figure 5A). According to waves (w), human scalp hair was further divided into four other types (Figure 5C), while the w of all pig hair types was less than $3 \mu \mathrm{m}$ (Figure 5B).

Taken together, Guard_2 showed higher similarity to type II human scalp hair while other pig hair types (Guard_1, Awl, Auchene, and Zigzag) were similar to type I of human 

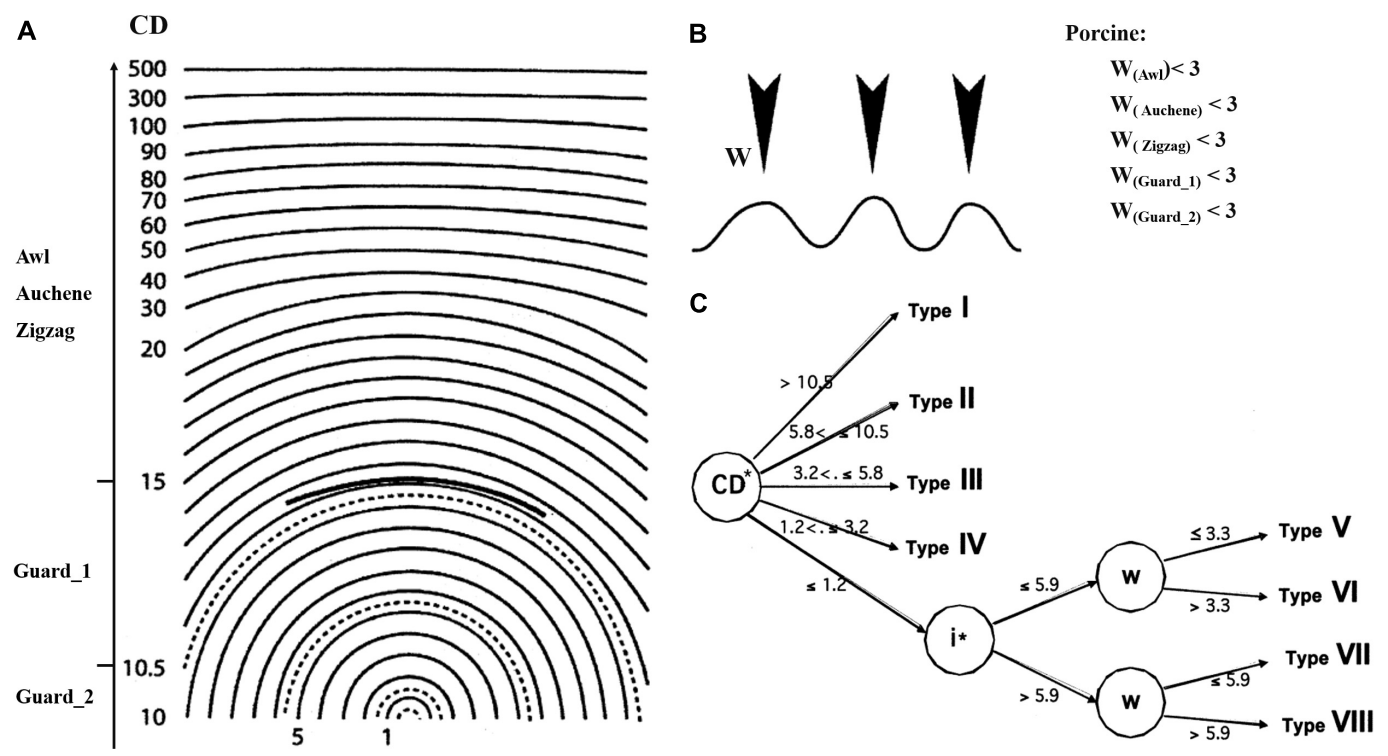

D Distribution of Hair Types in Country Groups

\begin{tabular}{|c|c|c|c|c|c|c|c|c|c|c|}
\hline & $\mathbf{N}$ & Type I (\%) & Type II (\%) & Type III (\%) & Type IV (\%) & Type V (\%) & Type VI (\%) & Type VII (\%) & Type VIII (\%) & Total (\%) \\
\hline South Africa & 141 & & & & 1 & 2 & 9 & 51 & 38 & 100 \\
\hline Ghana & 98 & & & & & 15 & 67 & 17 & 1 & 100 \\
\hline United States & 85 & & & 1 & 13 & 53 & 15 & 17 & 1 & 100 \\
\hline West Indies & 24 & & 4 & 8 & 34 & 46 & 8 & & & 100 \\
\hline North Africa & 133 & & 2 & 8 & 28 & 57 & 5 & & & 100 \\
\hline Brazil & 162 & 9 & 17 & 35 & 26 & 11 & 2 & & & 100 \\
\hline Mexico & 60 & 27 & 41 & 27 & 5 & & & & & 100 \\
\hline India & 84 & 15 & 35 & 44 & 6 & & & & & 100 \\
\hline Spain & 35 & 14 & 40 & 32 & 14 & & & & & 100 \\
\hline France & 35 & 23 & 26 & 43 & 8 & & & & & 100 \\
\hline United Kingdom & 58 & 10 & 33 & 38 & 19 & & & & & 100 \\
\hline Denmark & 47 & 17 & 40 & 32 & 11 & & & & & 100 \\
\hline Poland & 35 & 11 & 57 & 29 & 3 & & & & & 100 \\
\hline Germany & 99 & 23 & 35 & 27 & 15 & & & & & 100 \\
\hline Korea & 28 & 22 & 57 & 21 & & & & & & 100 \\
\hline Japan & 40 & 13 & 67 & 20 & & & & & & 100 \\
\hline Thailand & 65 & 19 & 46 & 32 & 3 & & & & & 100 \\
\hline China & 213 & 45 & 48 & 7 & & & & & & 100 \\
\hline
\end{tabular}

FIGURE 5 | The hair types between pig and human scalp hair. (A) CD meter used in the determination of five pig hair type curve diameter. (B) Number of waves (W) in five pig hair types. (C) Segmentation tree of eight human scalp hair; CD meter template derived from Bailey and Schliebe (1985); the graphics of the waves in (B) derived from De la Mettrie et al. (2007). (D) Distribution of hair types in country groups.

TABLE 2 | Hair characteristic parameters between human and pig.

\begin{tabular}{|c|c|c|c|c|c|c|}
\hline Species & Hair type & $\begin{array}{l}\text { Hair thickness } \\
\qquad(\mu \mathrm{m})^{\star}\end{array}$ & \multicolumn{3}{|c|}{ Hair density $\left(/ \mathrm{cm}^{2}\right)^{*}$} & References \\
\hline Pig & $\begin{array}{c}\text { Awl, Guard_1, Guard_2, } \\
\text { Auchene, Zigzag }\end{array}$ & $18-49$ & $46-53$ & $24-28$ & $13-27$ & Our study \\
\hline
\end{tabular}

*Hair thickness and hair density in human was based on scalp hair; ${ }^{1}$ human limb contains upper arm, forearm, thigh and calf.

scalp hair. More interestingly, the type I and II human scalp hair makes up a large proportion of eight different human scalp hair types, particularly for Asians and Europeans, and type II was in higher proportion than type I in all samples
(Figure 5D). However, types III-VIII of human scalp hair were not detected in our study mainly because only Yorkshire pig was used. Other pig breeds with curly/wool hair such as the Mangalica pig (Schachler et al., 2020), Turopolje pig 
(Čandek-PotokarRosa and Nieto, 2019), Canastrao pig (Rhoad, 1934), or Cuino pig (Lemus-Flores et al., 2005) could be used to identify more hair types. Thus, considering the similarity of the pig hair types to human scalp hair, pig proves to be an ideal model for human HF research.

In addition to hair types, pig and human hair also demonstrated similarity in hair thickness and hair density (Table 2). The hair thickness of pig $(18-49 \mu \mathrm{m})$ is closer to human (16-42 $\mu \mathrm{m})$ (Otberg et al., 2004) and the hair density of back $\left(24-28 / \mathrm{cm}^{2}\right)$ and limb $\left(13-27 / \mathrm{cm}^{2}\right)$ hair of pig in this study was very close to that of human back $\left(29 / \mathrm{cm}^{2}\right)$ and limb $\left(14-32 / \mathrm{cm}^{2}\right.$ ) (Otberg et al., 2004). Although the hair density of human scalp hair $\left(292 / \mathrm{cm}^{2}\right)$ was much higher than pig head (46$53 / \mathrm{cm}^{2}$ ), the trend of a higher head HF density than other body parts was shared by pig and human (Table 2 and Figure 1D). Unlike mice, which exhibit pelage with synchronous growth patterns, the asynchronous hair growth in different human body parts (Buffoli et al., 2014) leads to more growth of the HFs on the scalp and face than on the arms and legs (Seago and Ebling, 1985;
Pagnoni et al., 1994). Thus, based on our results which showed similarity of hair type and growth pattern between pig and human, it could be speculated that the pig hair growth is also asynchronous (Koch et al., 2020).

Based on 11 different embryonic days and 6 postnatal days, our results indicated that pig HF morphogenesis development started from E37 and ended at P45. According to eight key time points from E37-P45, we further summarized three periods of the HF morphogenesis development during embryogenesis, induction (E37-E41), organogenesis (E41-E85) and cytodifferentiation (>E85) (Figure 6).

Generally, prenatal HF morphogenesis development has been divided into three main periods. The morphological characteristics of hair placode, DC, DP, etc., at different periods are nearly the same in human (Muller et al., 1991), mice (Schneider et al., 2009), sheep (Zhang et al., 2019), rabbit (Ding et al., 2020) and, based on the current study, even in pig. However, the hair type and commence time points of each HF morphogenesis development stage has been shown to vary

\section{Pig Hair Follicle Morphogenesis}

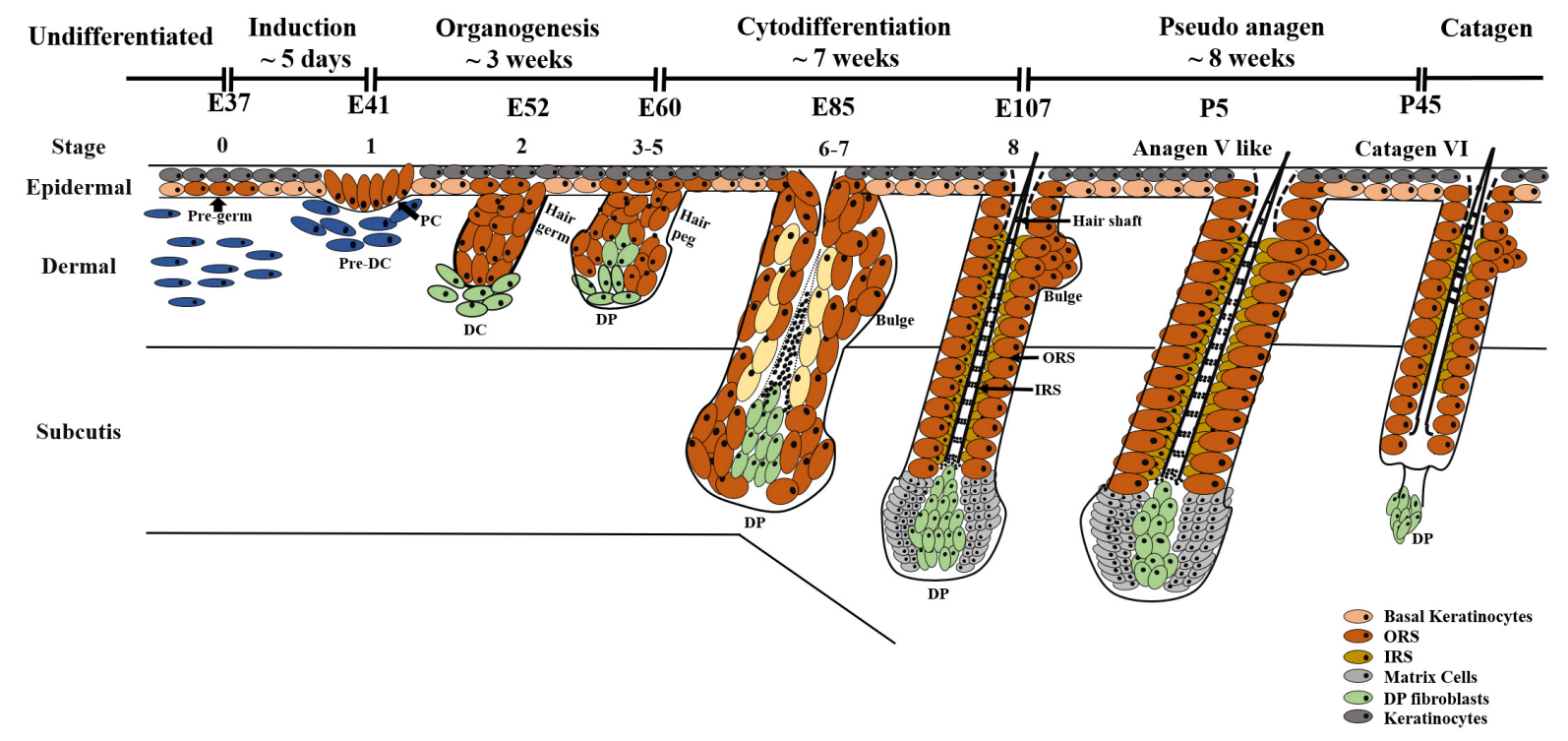

FIGURE 6 | HF morphogenesis development during pig embryogenesis. HF morphogenesis development in the prenatal stage is critically important, which determines HF cycle development in the postnatal stage. Pig HF morphogenesis development has been summarized into three main periods from E41 to P45 induction (E37-E41), organogenesis (E41-E85), and cytodifferentiation (>E85). Stages 1-8 of embryonic hair development are depicted, demonstrating the continuous transition between hair follicle development and the first postnatal hair cycle. PC, hair placode; DC, dermal condensate; DP, dermal papilla; IRS, inner root sheath; ORS, outer root sheath.

TABLE 3 | Classification of the hair follicle morphogenesis periods in different species.

\begin{tabular}{|c|c|c|c|c|c|c|c|c|}
\hline Species & Induction & Organogenesis & Cytodifferentiation & Mature hair & Pregnancy period & Hair visible in birth & HF starting stage* & References \\
\hline Human & 12th weeks & 14th weeks & 16th weeks & 18th weeks & 〜 40 weeks & Yes & Early/Mid & $\begin{array}{l}\text { Muller et al., 1991; } \\
\text { Chang et al., } 2005\end{array}$ \\
\hline Pig & 6th weeks & 7th weeks & 12th weeks & 15th weeks & $\sim 16$ weeks & Yes & Early/Mid & Our study \\
\hline Mouse & E13.5-14.5 & E15.5-17.5 & E18.5 & P3 & $\sim 21$ days & No & Mid/Late & $\begin{array}{c}\text { Duverger and } \\
\text { Morasso, } 2009\end{array}$ \\
\hline
\end{tabular}

*HF begins morphogenesis development in the embryo stages. Early, early gestational stage; Mid, middle gestational stage; Late, late gestational stage. 
largely, e.g., secondary HF in sheep and rabbit could make the HF morphogenesis complicated, thus making them not ideal model systems for human HF research.

As summarized in Table 3, fetal HF induction occurs in about the 12th week, organogenesis in the 14th week, cytodifferentiation in the 16th week, and mature hair in the 18th week of gestation (Muller et al., 1991). Comparing the average gestation period of human ( $\sim 40$ weeks), pig ( $\sim 16$ weeks), and mouse ( $\sim 21$ days), it can be concluded that prenatal HF morphogenesis development goes through in the early/mid gestational stage in human $(\sim 12$ weeks $)$ and pig $(\sim 6$ weeks), while in mouse, mid/late gestational stage occurs ( $\sim 14$ days). Moreover, hair shafts emerge through the epidermis before birth in both human and pig, while the mouse hair shaft is not visible until 1 week after birth (Harland, 2018). These differences are reflected consistently in human and mouse HF studies. According to the Duverger and Morasso (2009), the first HF cycle begins from the first catagen stage after birth. To explore the entire HF morphogenesis in pig, HF development in postnatal stages was also investigated in the current study. Our results showed that most HFs enter catagen at P45 while some are kept in pseudo anagen $\mathrm{V}$. This phenomenon might be a result of the asynchronous HF development of different hair types. Similar asynchronous HF development is also found in human; the normal ratio between active anagen hairs and resting telogen hairs is 85/15 in human scalp hair (Robertson, 1999) because of the asynchronous hair growth and development periods in different body regions. However, the different hair types in mouse coat were synchronous in growth (Koch et al., 2020). In the current study, we have only provided a rough time point for each of the HF morphogenesis development stages; further research is needed for a better understanding of the different time points in pig HF morphogenesis.

Over the past several decades, the pig has become an increasingly popular biomedical model especially in skin research. Both human and pig show abundant subdermal adipose tissue (Morris and Hopewell, 1990), similar regeneration time of epidermal cells (30 day for pig vs. 27-28 day for human) (Weinstein, 1965), and thickness of epidermis (30-140 nm for pig vs. 50-120 nm for human) (Meyer et al., 1978). The marker genes (Table 1) also showed consistent expression patterns in pig in comparison to human and mouse. Our previous study also shows that other classical pathway marker genes like Wnt5a, CTNNB1, DKK4, and BMP4 reported in mouse (Saxena et al., 2019) and human HF (Koch et al., 2020) were also being expressed in hair placode formation during the induction and organogenesis stages in pig (Jiang et al., 2019). Thus, collectively, our study demonstrates the shared similarities in hair types and HF morphogenesis development pattern between pig and human, further bolstering our hypothesis about the pig being an ideal model for HF research.

In summary, in this study, we explored the various hair types and classification of distinct stages of HF morphogenesis in pig by their morphology and marker gene expression. Our findings indicated that pig and human show a higher similarity in hair types and HF morphogenesis patterns. Thus, apart from the pig being a better model to decipher human HF morphogenesis, it also offers the opportunity to researchers of being a new model of hair research. Although in this study we pay more concern to the pig HF morphogenesis development in embryogenesis, it is also plausible to use pig as an ideal animal model to investigate human hair cycle after birth due to the similarity of pig hair types, gene expression patterns, and asynchronous growth patterns similar to human in postnatal stages, although further studies are necessary to confirm these aspects.

\section{DATA AVAILABILITY STATEMENT}

The original contributions presented in the study are included in the article/Supplementary Material, further inquiries can be directed to the corresponding author.

\section{ETHICS STATEMENT}

The animal study was reviewed and approved by the Institutional Animal Care and Use Committee of China Agricultural University.

\section{AUTHOR CONTRIBUTIONS}

XD and YJ: conceptualization. XD: methodology, writing, review, editing, and funding acquisition. YJ and QZ: formal analysis. SL and YW: investigation. QZ: visualization. BL and TL: resources. YJ: writing - original draft preparation. All authors contributed to the article and approved the submitted version.

\section{FUNDING}

This work was supported by grants from the National Natural Science Foundation of China (32070568 and 31671327), the National Key Research and Development Program (2019YFE0106800), and the China Agriculture Research System of MOF and MARA.

\section{ACKNOWLEDGMENTS}

The authors gratefully acknowledge their colleagues in the molecular quantitative genetics team of China Agricultural University for their helpful comments on the manuscript.

\section{SUPPLEMENTARY MATERIAL}

The Supplementary Material for this article can be found online at: https://www.frontiersin.org/articles/10.3389/fcell.2021. 721979/full\#supplementary-material

Supplementary Figure 1 | Specificity detection of five antibodies in pig hair follicle. FBS: negative control, fetal bovine serum substitute antibody. 
Supplementary Figure 2 | H\&E staining of Hair follicle morphogenesis at 11 different prenatal and six postnatal times (E37-P45). The red arrow represents the hair placode and hair germ. The green arrow represents the hair peg. The Blue

\section{REFERENCES}

Bailey, J., and Schliebe, S. (1985). "The precision of average curvature measurement," in Proceedings of the Symposium on Forensic Hair Comparisons, Federal Bureau of Investigation: Human Hair, Washington, DC: US Government Printing Office, 147-148.

Boras-Granic, K., Chang, H., Grosschedl, R., and Hamel, P. A. (2006). Lef1 is required for the transition of Wnt signaling from mesenchymal to epithelial cells in the mouse embryonic mammary gland. Dev. Biol. 295, 219-231. doi: 10.1016/j.ydbio.2006.03.030

Buffoli, B., Rinaldi, F., Labanca, M., Sorbellini, E., Trink, A., Guanziroli, E., et al. (2014). The human hair: from anatomy to physiology. Int. J. Dermatol. 53, 331-341. doi: 10.1111/ijd.12362

Čandek-PotokarRosa, M., and Nieto, N. (2019). European Local Pig Breeds Diversity and Performance A Study of Project TREASURE. London: IntechOpen.

Chang, C. H., Tsai, R. K., and Yu, H. S. (2005). Apoptosis coordinates with proliferation and differentiation during human hair follicle morphogenesis. J. Dermatol. Sci. 39, 9-16. doi: 10.1016/j.jdermsci.2005.01.014

De la Mettrie, R., Saint-Leger, D., Loussouarn, G., Garcel, A., Porter, C., and Langaney, A. (2007). Shape variability and classification of human hair: a worldwide approach. Hum. Biol. 79, 265-281. doi: 10.1353/hub.2007. 0045

Ding, H., Cheng, G., Leng, J., Yang, Y., Zhao, X., Wang, X., et al. (2020). Analysis of histological and microRNA profiles changes in rabbit skin development. Sci. Rep. 10:454. doi: 10.1038/s41598-019-57327-5

Duverger, O., and Morasso, M. I. (2009). Epidermal patterning and induction of different hair types during mouse embryonic development. Birth Defects Res. C Embryo Today 87, 263-272. doi: 10.1002/bdrc.20158

Duverger, O., and Morasso, M. I. (2014). To grow or not to grow: hair morphogenesis and human genetic hair disorders. Semin. Cell Dev. Biol. 25-26, 22-33. doi: 10.1016/j.semcdb.2013.12.006

Fantauzzo, K. A., Kurban, M., Levy, B., and Christiano, A. M. (2012). Trps1 and its target gene Sox9 regulate epithelial proliferation in the developing hair follicle and are associated with hypertrichosis. PLoS Genet. 8:e1003002. doi: 10.1371/journal.pgen.1003002

Fuchs, E. (2016). Epithelial skin biology: three decades of developmental biology, a hundred questions answered and a thousand new ones to address. Curr. Top. Dev. Biol. 116, 357-374. doi: 10.1016/bs.ctdb.2015.11.033

Gay, D. L., Yang, C. C., Plikus, M. V., Ito, M., Rivera, C., Treffeisen, E., et al. (2015). CD133 expression correlates with membrane beta-catenin and E-cadherin loss from human hair follicle placodes during morphogenesis. J. Invest. Dermatol. 135, 45-55. doi: 10.1038/jid.2014.292

Harland, D. P. (2018). Introduction to hair development. Adv. Exp. Med. Biol. 1054, 89-96. doi: 10.1007/978-981-10-8195-8_8

Jensen, T. W., Mazur, M. J., Pettigew, J. E., Perez-Mendoza, V. G., Zachary, J., and Schook, L. B. (2010). A cloned pig model for examining atherosclerosis induced by high fat, high cholesterol diets. Anim. Biotechnol. 21, 179-187. doi: 10.1080/10495398.2010.490693

Jiang, Y., Jiang, Y., Zhang, H., Mei, M., Song, H., Ma, X., et al. (2019). A mutation in MAP2 is associated with prenatal hair follicle density. FASEB J. 33, 14479-14490. doi: 10.1096/fj.201901187R

Koch, S. L., Tridico, S. R., Bernard, B. A., Shriver, M. D., and Jablonski, N. G. (2020). The biology of human hair: a multidisciplinary review. Am. J. Hum. Biol. 32:e23316. doi: 10.1002/ajhb.23316

Lemus-Flores, C., Morales, R. A., Haro, J. G. H., Alonso-Spilsbury, M., RamírezNecoechea, R., and Mota-Rojas, D. (2005). Growth, morphometry and reproductive performance of creole cuino pigs in Mexico. J. Anim. Vet. Adv. 4, 855-858.

Liu, J., Zhou, S., Li, S., Jiang, Y., Wan, Y., Ma, X., et al. (2019). Eleven genes associated with progression and prognosis of endometrial cancer (EC) identified by comprehensive bioinformatics analysis. Cancer Cell Int. 19:136. doi: 10.1186/s12935-019-0859-1

Liu, Y., Chen, J. Y., Shang, H. T., Liu, C. E., Wang, Y., Niu, R., et al. (2010). Light microscopic, electron microscopic, and immunohistochemical comparison arrow represents the (1) outer root sheath, ORS; (2) inner root sheath, IRS; (3) hair shaft; (4) dermal papilla, DP. The red dotted line represents the critical period of hair follicle morphogenesis development; Scale bars, $200 \mu \mathrm{m}$.

of Bama minipig (Sus scrofa domestica) and human skin. Comp. Med. 60, $142-148$.

Meurens, F., Summerfield, A., Nauwynck, H., Saif, L., and Gerdts, V. (2012). The pig: a model for human infectious diseases. Trends Microbiol. 20, 50-57. doi: 10.1016/j.tim.2011.11.002

Meyer, W., Schwarz, R., and Neurand, K. (1978). The skin of domestic mammals as a model for the human skin, with special reference to the domestic pig. Curr. Probl. Dermatol. 7, 39-52. doi: 10.1159/000401274

Miyachi, K., Yamada, T., Kawagishi-Hotta, M., Hasebe, Y., Date, Y., Hasegawa, S., et al. (2018). Extracellular proteoglycan decorin maintains human hair follicle stem cells. J. Dermatol. 45, 1403-1410. doi: 10.1111/1346-8138.1 4678

Morris, G. M., and Hopewell, J. W. (1990). Epidermal cell kinetics of the pig: a review. Cell Tissue Kinet. 23, 271-282. doi: 10.1111/j.1365-2184.1990.tb01124.x

Muller, M., Jasmin, J. R., Monteil, R. A., and Loubiere, R. (1991). Embryology of the hair follicle. Early Hum. Dev. 26, 159-166. doi: 10.1016/0378-3782(91)90155-v

Nguyen, M. B., Cohen, I., Kumar, V., Xu, Z., Bar, C., Dauber-Decker, K. L., et al. (2018). FGF signalling controls the specification of hair placode-derived SOX9 positive progenitors to Merkel cells. Nat. Commun. 9:2333. doi: 10.1038/ s41467-018-04399-y

Otberg, N., Richter, H., Schaefer, H., Blume-Peytavi, U., Sterry, W., and Lademann, J. (2004). Variations of hair follicle size and distribution in different body sites. J. Invest. Dermatol. 122, 14-19. doi: 10.1046/j.0022-202X.2003.22110.x

Pabst, R. (2020). The pig as a model for immunology research. Cell Tissue Res. 380, 287-304. doi: 10.1007/s00441-020-03206-9

Pagnoni, A., Kligman, A. M., El Gammal, S., and Stoudemayer, T. (1994). Determination of density of follicles on various regions of the face by cyanoacrylate biopsy: correlation with sebum output. Br. J. Dermatol. 131, 862-865. doi: 10.1111/j.1365-2133.1994.tb08590.x

Paus, R., Muller-Rover, S., Van Der Veen, C., Maurer, M., Eichmuller, S., Ling, G., et al. (1999). A comprehensive guide for the recognition and classification of distinct stages of hair follicle morphogenesis. J. Invest. Dermatol. 113, 523-532. doi: 10.1046/j.1523-1747.1999.00740.x

Porter, R. M. (2003). Mouse models for human hair loss disorders. J. Anat. 202, 125-131. doi: 10.1046/j.1469-7580.2003.00140.x

Pratt, C. H., King, L. E. Jr., Messenger, A. G., Christiano, A. M., and Sundberg, J. P. (2017). Alopecia areata. Nat. Rev. Dis. Primers 3:17011. doi: 10.1038/nrdp.20 17.11

Purba, T. S. I, Haslam, S., Shahmalak, A., Bhogal, R. K., and Paus, R. (2015). Mapping the expression of epithelial hair follicle stem cell-related transcription factors LHX2 and SOX9 in the human hair follicle. Exp. Dermatol. 24, 462-467. doi: 10.1111/exd.12700

Renner, S., Fehlings, C., Herbach, N., Hofmann, A., von Waldthausen, D. C., Kessler, B., et al. (2010). Glucose intolerance and reduced proliferation of pancreatic beta-cells in transgenic pigs with impaired glucose-dependent insulinotropic polypeptide function. Diabetes 59, 1228-1238. doi: 10.2337/ db09-0519

Rhoad, A. (1934). Woolly hair in swine. J. Hered. 25, 3-18.

Robertson, J. (1999). "Forensic and microscopic examination of human hair," in Forensic Examination of Hair, ed. J. Robertson (Abingdon-on-Thames: Taylor \& Francis), 79-154.

Rogers, C. S., Hao, Y., Rokhlina, T., Samuel, M., Stoltz, D. A., Li, Y., et al. (2008). Production of CFTR-null and CFTR-DeltaF508 heterozygous pigs by adenoassociated virus-mediated gene targeting and somatic cell nuclear transfer. J. Clin. Invest. 118, 1571-1577. doi: 10.1172/JCI34773

Ross, J. W., Fernandez de Castro, J. P., Zhao, J., Samuel, M., Walters, E., Rios, C., et al. (2012). Generation of an inbred miniature pig model of retinitis pigmentosa. Invest. Ophthalmol. Vis. Sci. 53, 501-507. doi: 10.1167/iovs.118784

Saxena, N., Mok, K. W., and Rendl, M. (2019). An updated classification of hair follicle morphogenesis. Exp. Dermatol. 28, 332-344. doi: 10.1111/exd.13913

Schachler, K., Distl, O., and Metzger, J. (2020). Tracing selection signatures in the pig genome gives evidence for selective pressures on a unique curly hair phenotype in Mangalitza. Sci. Rep. 10:22142. doi: 10.1038/s41598-020-79037-z 
Schneider, M. R., Schmidt-Ullrich, R., and Paus, R. (2009). The hair follicle as a dynamic miniorgan. Curr. Biol. 19, R132-R142. doi: 10.1016/j.cub.2008.12.005

Seago, S. V., and Ebling, F. J. (1985). The hair cycle on the human thigh and upper arm. Br. J. Dermatol. 113, 9-16. doi: 10.1111/j.1365-2133.1985.tb02038.x

Sennett, R., Wang, Z., Rezza, A., Grisanti, L., Roitershtein, N., Sicchio, C., et al. (2015). An integrated transcriptome atlas of embryonic hair follicle progenitors, their niche, and the developing skin. Dev. Cell 34, 577-591. doi: 10.1016/j. devcel.2015.06.023

Telerman, S. B., Rognoni, E., Sequeira, I., Pisco, A. O., Lichtenberger, B. M., Culley, O. J., et al. (2017). Dermal blimp1 acts downstream of epidermal TGFbeta and Wnt/beta-catenin to regulate hair follicle formation and growth. J. Invest. Dermatol. 137, 2270-2281. doi: 10.1016/j.jid.2017.06.015

Tomann, P., Paus, R., Millar, S. E., Scheidereit, C., and Schmidt-Ullrich, R. (2016). Lhx2 is a direct NF-kappaB target gene that promotes primary hair follicle placode down-growth. Development 143, 1512-1522. doi: 10.1242/dev.13 0898

Tsang, H. G., Rashdan, N. A., Whitelaw, C. B., Corcoran, B. M., Summers, K. M., and MacRae, V. E. (2016). Large animal models of cardiovascular disease. Cell Biochem. Funct. 34, 113-132. doi: 10.1002/cbf.3173

Turner, N. J., Pezzone, D., and Badylak, S. F. (2015). Regional variations in the histology of porcine skin. Tissue Eng. Part C Methods 21, 373-384. doi: 10.1089/ ten.TEC.2014.0246

van Ravenzwaay, B., and Leibold, E. (2004). The significance of in vitro rat skin absorption studies to human risk assessment. Toxicol In Vitro 18, 219-225. doi: $10.1016 /$ j.tiv.2003.08.002

Weinstein, G. D. (1965). Autoradiographic studies on turnover time and protein synthesis in pig epidermis. J. Invest. Dermatol. 44, 413-419. doi: 10.1038/jid. 1965.75
Yang, R., Zheng, Y., Burrows, M., Liu, S., Wei, Z., Nace, A., et al. (2014). Generation of folliculogenic human epithelial stem cells from induced pluripotent stem cells. Nat. Commun. 5:3071. doi: 10.1038/ncomms 4071

Zhang, Y., Wang, L., Li, Z., Chen, D., Han, W., Wu, Z., et al. (2019). Transcriptome profiling reveals transcriptional and alternative splicing regulation in the early embryonic development of hair follicles in the cashmere goat. Sci. Rep. 9:17735. doi: 10.1038/s41598-019-54315-7

Zuidema, D., and Sutovsky, P. (2020). The domestic pig as a model for the study of mitochondrial inheritance. Cell Tissue Res. 380, 263-271. doi: 10.1007/s00441019-03100-z

Conflict of Interest: The authors declare that the research was conducted in the absence of any commercial or financial relationships that could be construed as a potential conflict of interest.

Publisher's Note: All claims expressed in this article are solely those of the authors and do not necessarily represent those of their affiliated organizations, or those of the publisher, the editors and the reviewers. Any product that may be evaluated in this article, or claim that may be made by its manufacturer, is not guaranteed or endorsed by the publisher.

Copyright (C) 2021 Jiang, Zou, Liu, Li, Wang, Liu and Ding. This is an open-access article distributed under the terms of the Creative Commons Attribution License (CC BY). The use, distribution or reproduction in other forums is permitted, provided the original author(s) and the copyright owner(s) are credited and that the original publication in this journal is cited, in accordance with accepted academic practice. No use, distribution or reproduction is permitted which does not comply with these terms. 